\title{
Inhibition of multiple myeloma-derived exosomes uptake suppresses the functional response in bone marrow stromal cell
}

\author{
YONGJIANG ZHENG ${ }^{1 *}$, CHENGGONG TU $^{2 *}$, JINGWEN ZHANG ${ }^{1}$ and JINHENG WANG ${ }^{2}$ \\ ${ }^{1}$ Department of Hematology, The Third Affiliated Hospital of Sun Yat-Sen University, Guangzhou, Guangdong 510630; \\ ${ }^{2}$ Key Laboratory of Protein Modification and Degradation, School of Basic Medical Sciences, Affiliated Cancer Hospital \\ and Institute of Guangzhou Medical University, Guangzhou, Guangdong 511436, P.R. China
}

Received September 11, 2018; Accepted December 7, 2018

DOI: $10.3892 /$ ijo.2019.4685

\begin{abstract}
The communication between multiple myeloma (MM) cells and bone marrow stromal cells (BMSCs) serves a pivotal role in MM progression by supporting MM cell growth, proliferation and drug resistance. An exosomes-based endogenous transport system has been determined as a novel mechanism of this communication by revealing the capacity for exchange of functional components between cells. An exosomes transfer-mediated biological response in recipient cells is strongly determined by the detailed routes and mechanisms of exosomes internalization, which are diverse and can depend on surface molecules on the membrane of the vesicle and the recipient cell. Understanding the routes of exosomes uptake during MM cell-BMSC communication is of great importance for the development of blocking strategies beneficial for MM treatment. In the present study, fluorescently-labeled exosomes and pharmacological inhibitors, which are known to interfere with different internalization pathways, were used to characterize the cellular mechanisms involved in the uptake of MM cell-derived exosomes by BMSCs. MM cell-derived exosomes can promote BMSC viability and induce changes in multiple pro-survival and pro-proliferation pathways in BMSCs. As determined by flow cytometry and confocal microscopy, the uptake of MM cell-derived exosomes proceeded primarily through endocytosis, via special caveolin-dependent endocytosis, and partially through macropinocytosis and membrane fusion. Furthermore, treatment with endocytosis inhibitors suppressed the exosomes-induced changes in pathways in BMSCs. Collectively, these results indicate that
\end{abstract}

Correspondence to: Professor Jinheng Wang, Key Laboratory of Protein Modification and Degradation, School of Basic Medical Sciences, Affiliated Cancer Hospital and Institute of Guangzhou Medical University, Guangzhou, Guangdong 511436, P.R. China E-mail: wangjh89@gzhmu.edu.cn

${ }^{*}$ Contributed equally

Key words: exosomes, uptake, endocytosis, multiple myeloma, bone marrow stromal cell endocytosis is the primary route of internalization of MM cell-derived exosomes by BMSCs and indicate that inhibition of exosomes uptake can interrupt the communication between MM cells and BMSCs and thus serve as a potential adjunctive strategy for MM treatment.

\section{Introduction}

Extracellular vesicles (EVs) represent a series of spherical membranous particles that are secreted from cells into the extracellular microenvironment (1). EVs enclose and transfer molecular cargoes, including proteins, nucleic acids, lipids and other metabolites, derived from the donor cells and deliver them to recipient cells (2). Based on intracellular origin and size, EVs are frequently classified into three clusters, termed apoptotic bodies, microvesicles and exosomes (3). Exosomes are small membranous vesicles between 30-150 $\mathrm{nm}$ in diameter, and are derived from late endosomes and multivesicular bodies (4). Exosomes function as a cell-to-cell communicator by naturally transferring macromolecules from producer to recipient cells (1). This communication system consists of multiple steps, including an exosomes release, local or long range transport, and exosomes capture and internalization, and finally results in the changes of recipient cell behavior, and modification of physiological and pathological processes (5-7). Exosomes uptake by target cells involves a variety of mechanisms, including clathrin- or caveolin-dependent endocytosis, macropinocytosis, phagocytosis, lipid raft-mediated internalization and membrane fusion $(8,9)$. Routes and mechanisms of exosomes uptake largely depend on surface molecules and glycoproteins on the membrane of the vesicle and the recipient cell, and normally, $>1$ route is involved in the internalization of exosomes (8). A number of pharmacological inhibitors, including heparin, wortmannin, dynasore, chlorpromazine, amiloride and omeprazole, have been frequently used to block an uptake route of specific exosomes (8). Heparin blocks the binding of heparin sulphate proteoglycans, which are exhibited on membrane particles and lipoproteins, to the plasma membrane, and thus inhibits endocytosis (10). Dynasore is a specific inhibitor of dynamin 2, which is required for clathrin-mediated and caveolin-dependent endocytosis (11). Wortmannin inhibits membrane insertion by suppressing phosphoinositide 
3-kinases (PI3Ks), eventually resulting in the inhibition of phagocytic processes, including phagosomes formation (12). Chlorpromazine can inhibit the formation of clathrin-coated pits by targeting multiple receptors, including dopamine, serotonin and histamine receptors, and thus serves as an inhibitor of clathrin-mediated endocytosis $(8,13)$. Amiloride, an inhibitor targeting the sodium/proton exchanger, is known to specifically inhibit macropinosome formation by reducing submembranous $\mathrm{pH}$ and preventing Rac family small GTPase 1/cell division cycle 42 signaling $(14,15)$. Omeprazole, a proton pump inhibitor, has been demonstrated to inhibit the fusion of the EV membrane with the cell plasma membrane by inducing both acidification of the cell cytosol and acidic vesicle retention within the cells (16). The intrinsic properties, including low toxicity and immunogenicity, high biocompatibility and biodegradability, and nanometric size, make exosomes highly suitable as drug carriers $(17,18)$, and identification of specific routes for their uptake will enable researchers to develop a more effective delivery system.

Multiple myeloma (MM), also known as plasma cell myeloma, is a hematological cancer that starts with the accumulation of uncontrolled monoclonal plasma cells in bone marrow (BM) (19). During MM progression, MM cells interact with other BM-derived cells, particularly with BM stromal cells (BMSCs), to support their own survival and growth (20). The interplay between them performs a crucial function in MM pathogenesis and this interaction is mediated by direct cell-to-cell contacts, soluble factors and exosomes (21). Additionally, the interaction of MM cell-derived exosomes with the bystander cells in the BM microenvironment enhances angiogenesis and immunosuppression, which favors MM progression $(22,23)$. The positive roles of exosomes-mediated communication between BMSCs and MM cells in MM progression was demonstrated by indicating that BMSC-derived exosomes promote MM cell survival, proliferation, migration and even drug resistance, and that MM cell-derived exosomes enhance pro-tumoral activity of BMSCs through the delivery of microRNAs (miRNAs) $(24,25)$. Judging by the important functions of exosomes in MM cell-BMSC communication, blockade of this mediator may facilitate MM treatment. Nonetheless, the detailed mechanisms involved in exosomes-mediated communication, particularly in exosomes internalization, remain unknown.

The routes and mechanisms of exosomes uptake strongly determine the ability of exosomes to transport their content and to elicit a biological response in recipient cells $(26,27)$. In the present study, the endocytic routes of uptake of MM cell-derived exosomes by BMSCs were determined and the effects of specific uptake inhibitors on MM exosomes-induced changes in BMSCs were assessed.

\section{Materials and methods}

Materials and reagents. Human MM cell lines H929, MM1S, RPMI8226 and U266 were purchased from China Center for Type Culture Collection (Wuhan, China). Human BM was collected for BMSC culture from a healthy donor (41 years old; male) in December 2017 at the Department of Hematology, The Third Affiliated Hospital of Sun Yat-Sen University after informed consent was obtained. Ethical approval was obtained from Ethical Committee for Clinical Medicine Research of The Third Affiliated Hospital of Sun Yat-Sen University in Guangzhou (Guangzhou, China). Primary antibodies against flotillin-1 (cat. no. 18634T; 1:1,000), calreticulin (cat. no. 12238T; 1:1,000), heat shock protein 70 (HSP70; cat. no. 4872T; 1:1,000), GAPDH (cat. no. 5174S; 1:1,000), Alix (cat. no. 2171S; 1:1,000), phosphor-(p-) extracellular signal-regulated kinase 1/2 (ERK1/2; cat. no. 4370T; 1:1,000), ERK1/2 (cat. no. 4695T; 1:1,000), p-c-Jun N-terminal kinase (JNK; cat. no. 9255S; 1:1,000), JNK2 (cat. no. 9258S; 1:1,000), p-signal transducer and activator of transcription 1 (STAT1; cat. no. 7649T; 1:1,000), STAT1 (cat. no. 9172T; 1:1,000), p-STAT3 (cat. no. 9138S; 1:1,000) and STAT3 (cat. no. 4904S; 1:1,000), as well as a horseradish peroxidase (HRP)-conjugated anti-mouse IgG antibody (cat. no. 7076S; 1:2,000) and HRP-conjugated anti-rabbit IgG antibody (cat. no. 7074S; 1:2,000), were purchased from Cell Signaling Technology, Inc. (Danvers, MA, USA). Anti-human cluster of differentiation 63 (CD63; cat. no. 10628D; 1:250) and antitumor susceptibility 101 (TSG101; cat. no. sc-7964; 1:200) antibodies were purchased from Thermo Fisher Scientific, Inc. (Waltham, MA, USA) and Santa Cruz Biotechnology, Inc. (Dallas, TX, USA), respectively. For inhibition experiments, heparin, wortmannin, dynasore, chlorpromazine, amiloride, and omeprazole were purchased from Selleck Chemicals (Houston, TX, USA).

Cell culture. H929, MM1S and RPMI8226 cells were maintained in RPMI-1640 (Gibco; Thermo Fisher Scientific, Inc.) supplemented with $10 \%$ fetal bovine serum (FBS; Excell Bio, Shanghai, China). U266 cells were cultured in RPMI-1640 supplemented with $15 \%$ of FBS. All these four MM cell lines were used for all experiments and cultured at $37^{\circ} \mathrm{C}$. The BM mononuclear cells were isolated via a Lymphoprep (Stemcell Technologies, Inc., Vancouver, BC, Canada) gradient and were cultured in OriCell Human MSC Culture medium (Cyagen Biosciences, Inc., Santa Clara, CA, USA) at $37^{\circ} \mathrm{C}$. After $24 \mathrm{~h}$, non-adherent cells were removed and adherent cells were continuously cultured in OriCell Human MSC Culture medium. Human BMSCs were used within 5 passages. All culture media were supplemented with $100 \mathrm{U} / \mathrm{ml}$ penicillin and $0.1 \mathrm{mg} / \mathrm{ml}$ streptomycin.

Exosomes isolation, purification, quantification and nanoparticle tracking analysis. Exosomes were isolated as described previously (24). MM cells were cultured in the serum-free RPMI-1640 medium at $37^{\circ} \mathrm{C}$ for $24 \mathrm{~h}$ and the conditioned medium (CM) was collected and then centrifuged for $5 \mathrm{~min}$ at 2,000 $\mathrm{x}$ g at room temperature to remove cells and large debris. Subsequently, CM was passed through $0.22 \mu \mathrm{m}$ filters and concentrated to $2 \mathrm{ml}$ in a 100K MWCO Advance Centrifugal Device (Pall Corporation, New York, NY, USA) for enriching exosomes. Concentrated CM was washed with $10 \mathrm{ml}$ PBS once using a concentrator and passed again through a $0.22 \mu \mathrm{m}$ filter. Exosomes were precipitated with the ExoQuick-TC exosomes precipitation solution (System Biosciences, Palo Alto, CA, USA). The exosomes were resuspended in a serum-free RPMI-1640 medium, and the protein concentration was quantified with a Bicinchoninic Acid (BCA) Protein Assay kit (Thermo Fisher Scientific, Inc.). Particle size distribution of isolated exosomes was determined 
via a Zetaview Nanoparticle Tracking Analyzer (Particle Metrix GmbH, Meerbusch, Germany).

Western blot analysis. Cells and exosomes were lysed in radioimmunoprecipitation assay buffer (Beyotime Institute of Biotechnology, Haimen, China) and mixed with the Protease Inhibitor Cocktail (Beyotime Institute of Biotechnology). Protein concentrations were detected with a BCA Protein Assay kit. Lysates were mixed with loading buffer (Beyotime Institute of Biotechnology) and then heated to $95^{\circ} \mathrm{C}$ for $10 \mathrm{~min}$. Every sample was loaded onto 8 (for STAT1 detection) or $10 \%$ (for other protein detection) polyacrylamide gels (Beyotime Institute of Biotechnology) and subjected to electrophoresis. Subsequently, the proteins were transferred to polyvinylidene fluoride membranes (Beyotime Institute of Biotechnology), and the membrane was blocked in $5 \%$ non-fat milk for $1 \mathrm{~h}$ at room temperature. It has been demonstrated that non-fat milk did not affect the phosphorylation levels of the target proteins, including STAT1, STAT3 and ERK1/2, analyzed in the present study, as indicated in other studies (28-30). The membranes were incubated with primary antibodies for $2 \mathrm{~h}$ at room temperature and then with secondary antibodies for $2 \mathrm{~h}$ at room temperature. Protein bands were visualized and documented using an Enhanced Chemiluminescent Western Blotting solution (Beyotime Institute of Biotechnology) and GelView 6000 Pro (Guangzhou Biolight Equipment Co., Ltd., Guangzhou, China), respectively.

Cell viability assays. Human BMSCs were seeded at a density of 2,000 cells/well in a 96-well plate and cultured in OriCell Human MSC Culture medium at $37^{\circ} \mathrm{C}$. After $24 \mathrm{~h}$, the medium was replaced with non-serum Dulbecco's modified Eagle's medium (DMEM)/F12 (Gibco; Thermo Fisher Scientific, Inc.) at $37^{\circ} \mathrm{C}$ overnight. Exosomes and/or inhibitors, including heparin, wortmannin, dynasore, chlorpromazine, amiloride and omeprazole were added to BMSCs for 4 or $48 \mathrm{~h}$ at $37^{\circ} \mathrm{C}$. Cell viability was measured and recorded using a Cell Titer Glo Luminescent Viability Assay kit (Promega Corporation, Madison, WI, USA) and a Varioskan Flash Multimode Reader (Thermo Fisher Scientific, Inc.).

Labeling of exosomes with DIO. Membrane fluorescent dye DIO (Beyotime Institute of Biotechnology) was added to a H929 exosomes suspension at a final concentration of $10 \mu \mathrm{M}$ and was incubated for $30 \mathrm{~min}$ at $37^{\circ} \mathrm{C}$. An Exosome Spin Column (Thermo Fisher Scientific, Inc.) was employed to remove free DIO. Subsequently, $10 \mu \mathrm{M}$ DIO solutions were also processed via an Exosome Spin Column, and the flow-through served as a DIO control.

Confocal microscopy analysis. Human BMSCs were cultured in 24-well plates at a density of 50,000 cells/well in OriCell Human MSC Culture medium at $37^{\circ} \mathrm{C}$. DIO-labeled H929 exosomes and/or inhibitors were added to BMSCs for $4 \mathrm{~h}$ at 37 or $4^{\circ} \mathrm{C}$. Subsequently, the medium was discarded, and the cells were washed once with $1 \mathrm{ml}$ serum-free DMEM/F12 medium to remove excess exosomes. For labeling the cell nucleuses and lysosomes, Hoechst 33342 (Beyotime Institute of Biotechnology) and Lyso-Tracker Red (Beyotime Institute of Biotechnology) were added and incubated with human
BMSCs for $1 \mathrm{~h}$ at $37^{\circ} \mathrm{C}$. Fluorescent images were obtained using a TCS SP8 confocal microscope at x200 magnification (Leica Microsystems GmbH, Wetzlar, Germany).

Flow cytometry analysis. Following incubation with DIO-labeled H929 exosomes and/or inhibitors, BMSCs were trypsinized and collected. The mean and median fluorescent intensity in these cells were measured on a BD Accuri C6 cytometer (BD Biosciences; Becton Dickinson and Company, Franklin Lakes, NJ, USA) and analyzed using FlowJo V10 software (FlowJo LLC, Ashland, OR, USA).

Statistical analysis. GraphPad Prism 5 software (GraphPad Software, Inc., La Jolla, CA, USA) was employed to analyze the results and generate histograms presenting the data as the mean values \pm standard deviation. One-way analysis of variance with Tukey's post hoc test was performed to determine the statistical significance of differences between groups. $\mathrm{P}<0.05$ was considered to indicate a statistically significant difference.

\section{Results}

MM cell-derived exosomes increase BMSC viability. MM cell-derived exosomes were first isolated from the CM of four human MM cell lines, RPMI8226, H929, MM1S and U266. The sizes of exosomes were determined by nanoparticle tracking analysis, and a size distribution of 50-200 $\mathrm{nm}$ for the four types of exosomes was observed (Fig. 1A). A number of well-established markers of exosomes, including HSP70, Alix, TSG101, CD63 and flotillin-1 were detected in these exosomes, whereas an intracellular contaminant, calreticulin, was absent (Fig. 1B). To assess the effect of MM cell-derived exosomes on BMSC viability, BMSCs were cultured with exosomes isolated from the four cell lines at different final concentrations. These exosomes dose-dependently promoted cell viability (Fig. 1C). Additionally, MM cell-derived exosomes increased the phosphorylation of STAT1, STAT3 and ERK1/2, while phosphorylation of JNK was increased only slightly (Fig. 1D), indicating that these exosomes can affect multiple pathways in BMSCs.

Uptake of MM cell-derived exosomes by BMSCs is energy-dependent. To determine internalization of exosomes by BMSCs, the green dye DIO was used to fluorescently label the membrane of purified MM cell-derived exosomes, and BMSCs were visualized by staining lysosomes with Lyso-Tracker Red. Following incubation with labeled exosomes, an increasing green fluorescent signal was observed in BMSCs over time, as revealed by confocal microscopy analysis (Fig. 2A) and flow cytometry (Fig. 2B). At $4 \mathrm{~h}$ after incubation, internalization of a large number of exosomes was observed in almost all BMSCs (Fig. 2A). To test whether the uptake of exosomes by BMSCs is an active process or passive membrane fusion, BMSCs were incubated with labeled exosomes at $4^{\circ} \mathrm{C}$ for $4 \mathrm{~h}$. Internalization of $\mathrm{MM}$ cell-derived exosomes was inhibited almost completely at $4^{\circ} \mathrm{C}$ (Fig. $2 \mathrm{C}$ and D), indicating that this internalization is mediated by active endocytic processes and is energy-dependent.

The endocytosis inhibitor decreases internalization of MM cell-derived exosomes. Exosomes deliver a cargo and affect 
A

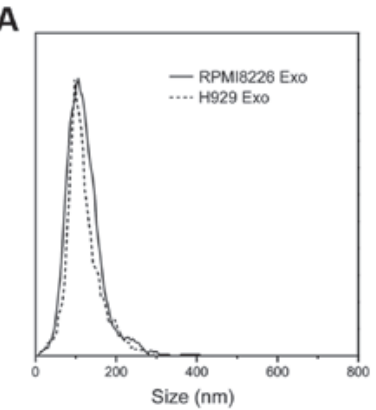

C
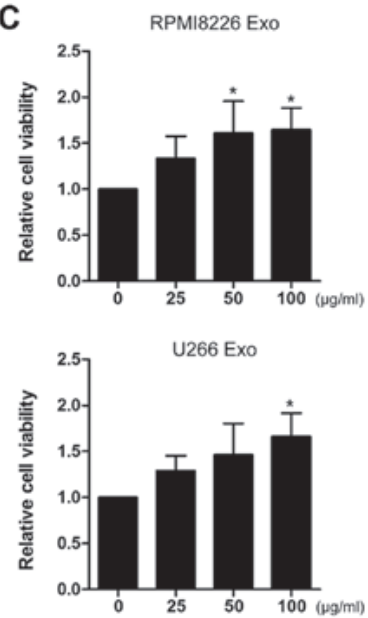

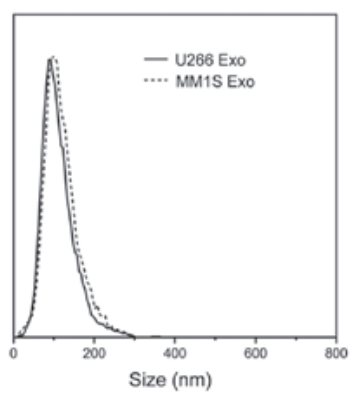

H929 Exo
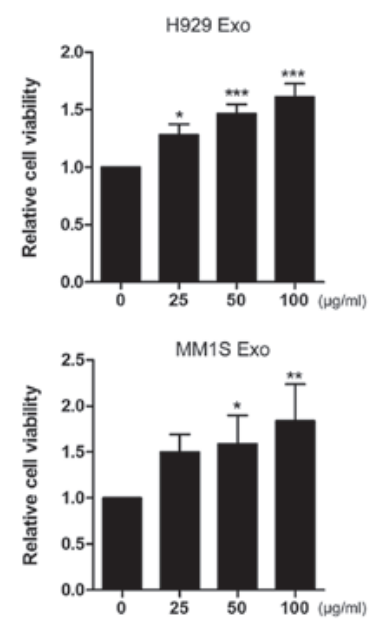

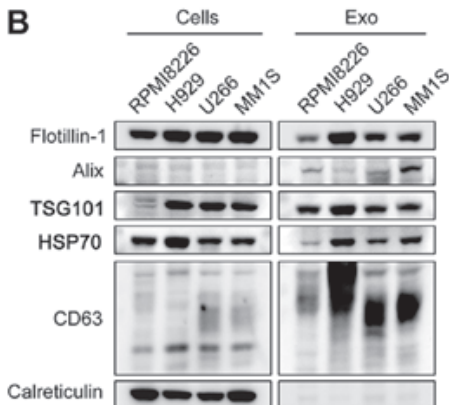

D

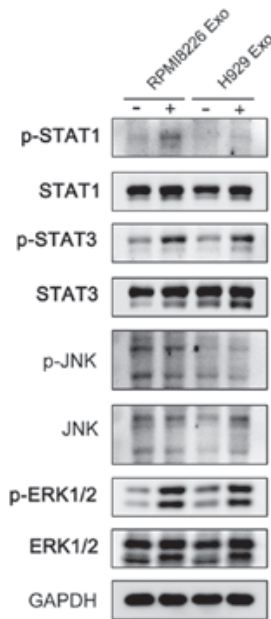

Figure 1. Multiple myeloma cell-derived exosomes increase human BMSC viability and activate multiple pathways. (A) Size distribution of exosomes isolated from RPMI8226, H929, U266 and MM1S cell lines was determined by nanoparticle tracking analysis. (B) Western blot analysis of exosomes-specific markers flotillin-1, Alix, TSG101, HSP70 and CD63, as well as a negative marker (calreticulin), in RPMI8226, H929, U266 and MM1S cell lysates and their exosomes (C) Human BMSCs in serum-free RPMI-1640 medium were treated with RPMI8226, H929, U266 or MM1S cell-derived exosomes at a concentration of 25,50 or $100 \mu \mathrm{g} / \mathrm{ml}$ for $48 \mathrm{~h}$, and then the cell viability was determined. The fold changes were measured by comparing the viability of exosomes-treated cells with that of untreated cells. (D) Human BMSCs were incubated with $100 \mu \mathrm{g} / \mathrm{ml}$ RPMI8226 or H929-derived exosomes for 6 h; subsequently, total and phosphorylated proteins STAT1, STAT3, JNK and ERK1/2 were quantified by western blot analysis. The GAPDH protein served as a loading control. Mean values \pm standard deviation for 3 independent experiments are depicted. ${ }^{*} \mathrm{P}<0.05,{ }^{* *} \mathrm{P}<0.01$ and ${ }^{* * *} \mathrm{P}<0.001$, compared with the control group. BMSCs, bone marrow stromal cells; Exo, exosomes; Cells, cell lysates; p, phosphor; STAT, signal transducer and activator of transcription 1; JNK, c-Jun N-terminal kinase; ERK1/2, extracellular signal-regulated kinase 1/2; CD63, cluster of differentiation; TSG101, tumor susceptibility 101; HSP70, heat shock protein 70.

target cells through membrane fusion, endocytosis and macropinocytosis. To identify the pathway involved in the uptake of MM cell-derived exosomes by BMSCs, heparin, an endocytosis inhibitor (10), and wortmannin, a phagocytosis blocker (31), were employed. In the presence of heparin, the internalization of MM cell-derived exosomes was notably suppressed (Fig. 3A), and the mean fluorescence intensity was significantly decreased by $\sim 78 \%$ in the presence of $20 \mu \mathrm{g} / \mathrm{ml}$ heparin (Fig. 3B), pointing to the involvement of endocytic mechanisms requiring cytoskeletal remodeling. In contrast, notable inhibition was not observed in the presence of wortmannin (Fig. 3C), and the mean and median fluorescence intensity underwent a slight decrease when the highest concentration of wortmannin was applied (Fig. 3D). Although the mean fluorescence intensity decreased by $30 \%$ in the presence of $0.5 \mu \mathrm{M}$ wortmannin, the median value did not change significantly. Since wortmannin primarily targets PI3Ks, these data indicated that internalization of MM cell-derived exosomes is independent of PI3K-involved macropinocytosis.

Internalization of MM cell-derived exosomes is independent of clathrin-dependent endocytosis. To identify the specific endocytic processes that contribute to the internalization of MM cell-derived exosomes, BMSCs were treated with pharmacological inhibitors known to interfere with clathrinor caveolin-dependent endocytosis. Dynamin 2 is a GTPase required for clathrin- and caveolin-dependent endocytosis (11), and its specific inhibitor, dynasore, notably reduced the internalization of exosomes in the present study even at the lowestconcentration $(25 \mu \mathrm{M})$ applied (Fig.4A). Nevertheless this inhibition was not dose-dependent (Fig. 4B). Chlorpromazine, an inhibitor of clathrin-mediated endocytosis (13), did not induce significant decreases in the exosomes uptake by BMSCs except for $1.25 \mu \mathrm{M}$ chlorpromazine that decreased the mean exosomes uptake by $20 \%$, as revealed by confocal microscopy (Fig. 4C) and flow cytometry (Fig. 4D). These data indicated that caveolin-dependent endocytosis is the primary endocytic process that contributes to the uptake of MM cell-derived exosomes by BMSCs.

Macropinocytosis and membrane fusion are partially involved in the uptake of MM cell-derived exosomes. Other pathways, including macropinocytosis and membrane fusion, are also routes of EV uptake, and thus, the effects of their inhibitors on the uptake of MM cell-derived exosomes by BMSCs were determined. An inhibitor targeting the 

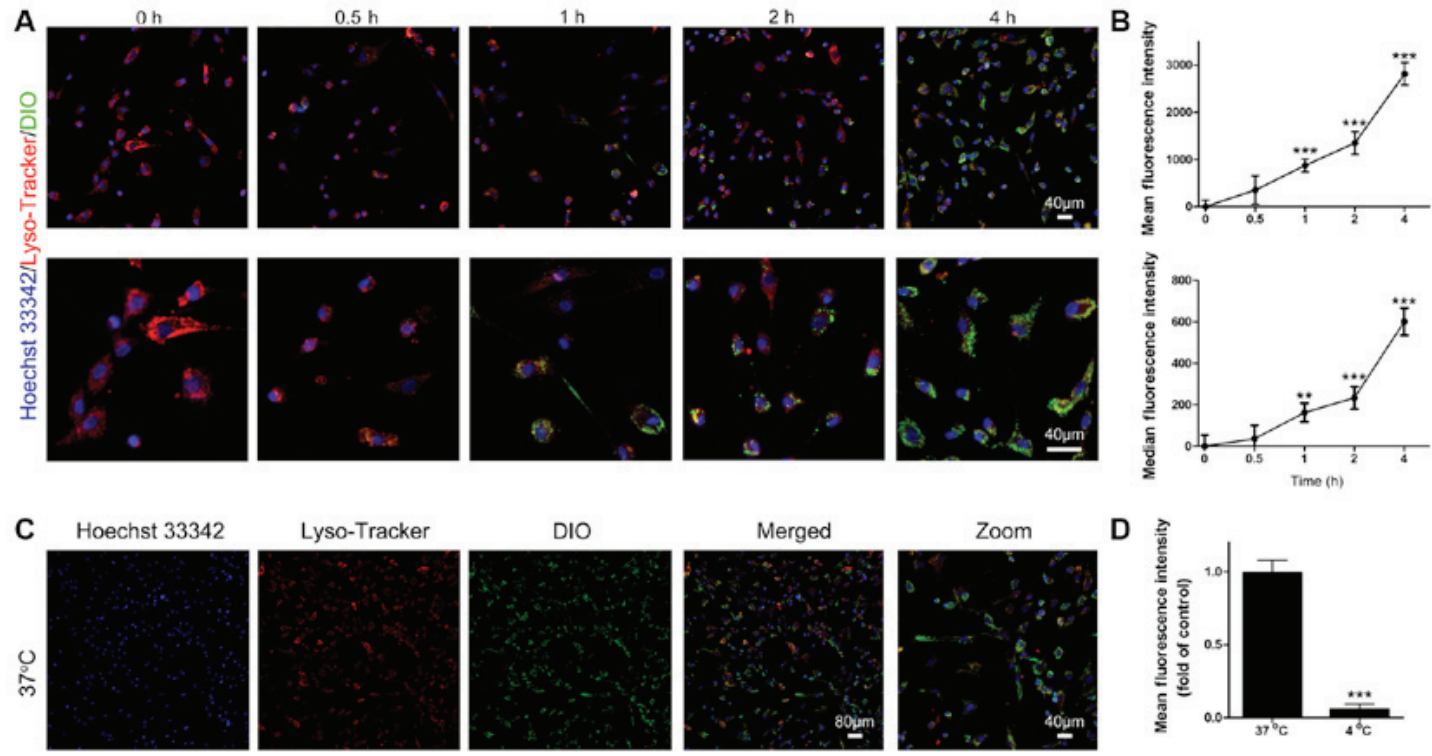

Lyso-Tracker

DIO
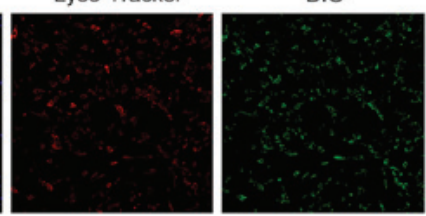

Merged

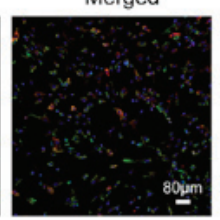

Zoom
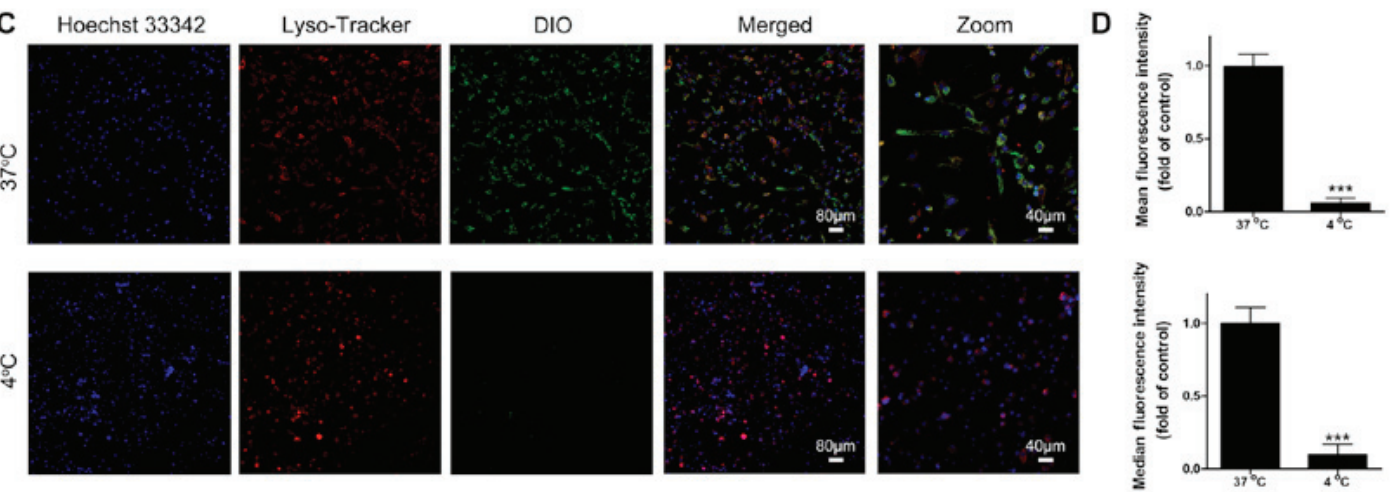

Figure 2. Uptake of multiple myeloma cell-derived exosomes by BMSCs is energy-dependent. Human BMSCs were cultured with DIO-labeled H929 cell-derived exosomes $(50 \mu \mathrm{g} / \mathrm{ml})$ for the indicated periods and the uptake was determined by (A) confocal microscopy and (B) quantified by flow cytometry. ${ }^{* *} \mathrm{P}<0.01 ;{ }^{* * * *} \mathrm{P}<0.001$, compared with $0 \mathrm{~h}$. Human BMSCs were cultured with DIO-labeled H929 cell-derived exosomes $(50 \mu \mathrm{g} / \mathrm{ml})$ at $37^{\circ} \mathrm{C}$ or $4^{\circ} \mathrm{C}$ for $4 \mathrm{~h}$ and the uptake was visualized by (C) confocal microscopy. (D) Mean or median fluorescence intensity was measured by flow cytometry. ${ }^{* * *} \mathrm{P}<0.001$, compared with the cells cultured at $37^{\circ} \mathrm{C}$. BMSCs, bone marrow stromal cells.

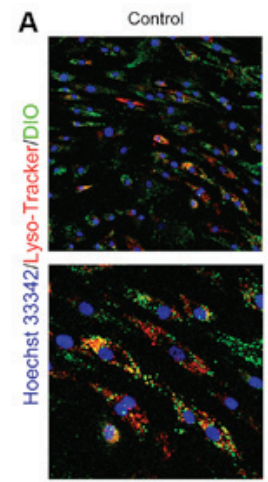

C
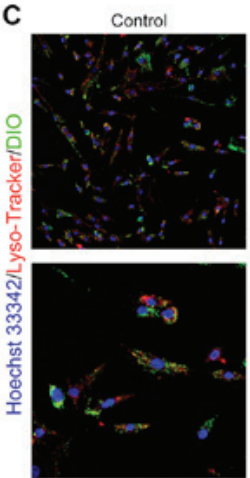

Heparin $(5 \mu g / m l)$

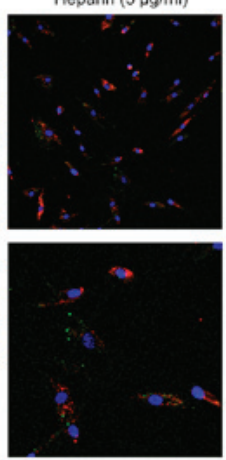

Wortmannin $(0.25 \mu \mathrm{M})$
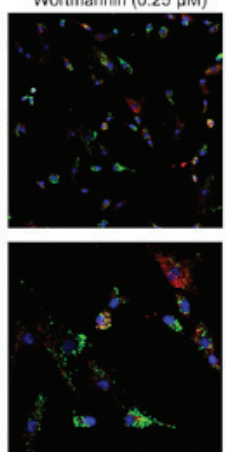

Heparin $(10 \mu \mathrm{g} / \mathrm{ml})$
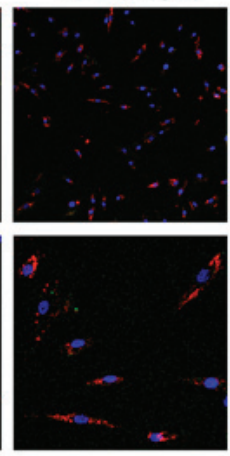

Wortmannin $(0.5 \mu \mathrm{M})$
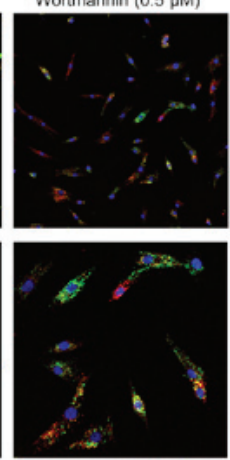

Heparin $(20 \mu g / m l)$
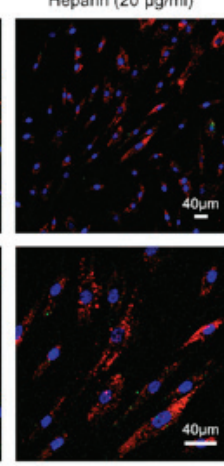

Wortmannin $(1 \mu M)$
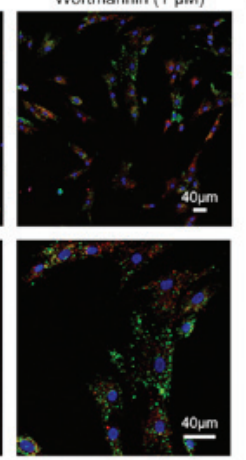
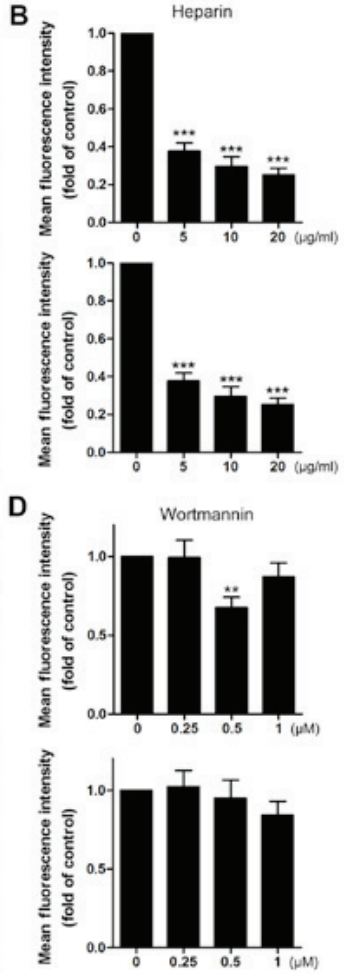

Figure 3. An endocytosis inhibitor reduces internalization of multiple myeloma cell-derived exosomes. Human BMSCs were pre-treated with the indicated concentrations of heparin for $0.5 \mathrm{~h}$ and then incubated with $50 \mu \mathrm{g} / \mathrm{ml}$ DIO-labeled $\mathrm{H} 929$ cell-derived exosomes for another $4 \mathrm{~h}$ in the presence of the indicated inhibitors. (A) The uptake was visualized by confocal microscopy and (B) the mean or median fluorescence intensity was measured by flow cytometry. Human BMSCs were pre-treated with the indicated concentrations of wortmannin for $0.5 \mathrm{~h}$ and then incubated with $50 \mu \mathrm{g} / \mathrm{ml} \mathrm{DIO}-\mathrm{labeled} \mathrm{H} 929 \mathrm{cell}-\mathrm{derived}$ exosomes for another $4 \mathrm{~h}$ in the presence of the indicated inhibitors. (C) The uptake was visualized by confocal microscopy and (D) the mean or median fluorescence intensity was measured by flow cytometry. ${ }^{* *} \mathrm{P}<0.01$ and ${ }^{* * * *} \mathrm{P}<0.001$, compared with the control group. BMSCs, bone marrow stromal cells. 

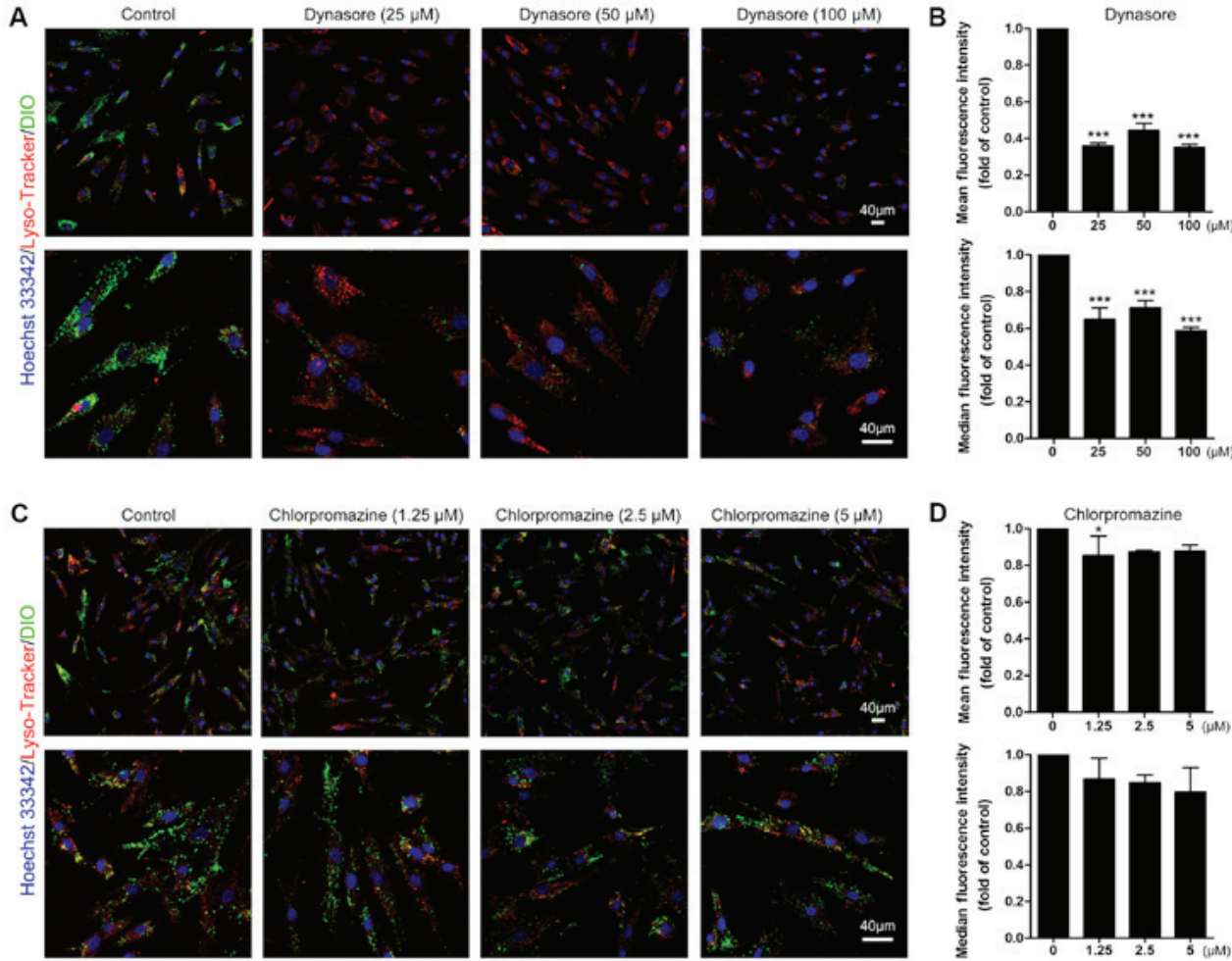

Figure 4. Internalization of multiple myeloma cell-derived exosomes does not involve clathrin-dependent endocytosis. Human BMSCs were pre-treated with the indicated concentration of dynasore for $0.5 \mathrm{~h}$ and then incubated with DIO-labeled $\mathrm{H} 929$ cell-derived exosomes $(50 \mu \mathrm{g} / \mathrm{ml})$ for another $4 \mathrm{~h}$ in the presence of the indicated inhibitors. (A) The uptake was visualized by confocal microscopy and (B) the mean or median fluorescence intensity was measured by flow cytometry. Human BMSCs were pre-treated with the indicated concentration of chlorpromazine for $0.5 \mathrm{~h}$ and then incubated with DIO-labeled H929 cell-derived exosomes $(50 \mu \mathrm{g} / \mathrm{ml})$ for another $4 \mathrm{~h}$ in the presence of the indicated inhibitors. (C) The uptake was visualized by confocal microscopy and (D) the mean or median fluorescence intensity was measured by flow cytometry. ${ }^{*} \mathrm{P}<0.05$ and ${ }^{* * *} \mathrm{P}<0.001$, compared with the control group. BMSCs, bone marrow stromal cells.
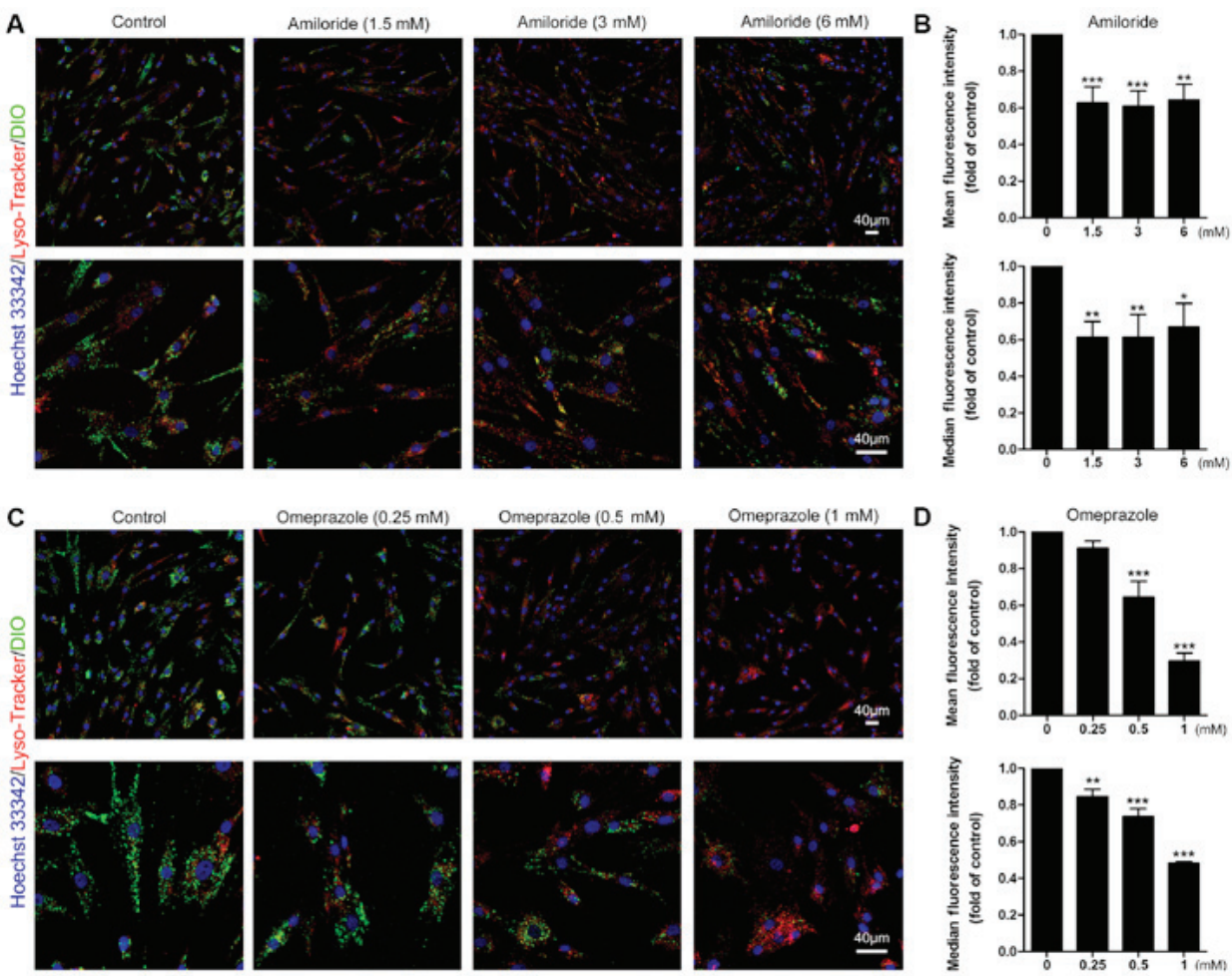

Figure 5. Inhibitors of macropinocytosis and membrane fusion partially decrease the uptake of multiple myeloma cell-derived exosomes. Human bone marrow stromal cells were pre-treated with the indicated concentration of amiloride for $0.5 \mathrm{~h}$ and then incubated with $50 \mu \mathrm{g} / \mathrm{ml}$ DIO-labeled H929 cell-derived exosomes for another $4 \mathrm{~h}$ in the presence of the indicated inhibitors. (A) The uptake was visualized by confocal microscopy and (B) the mean or median fluorescence intensity was determined by flow cytometry. Human bone marrow stromal cells were pre-treated with the indicated concentration of omeprazole for $0.5 \mathrm{~h}$ and then incubated with $50 \mu \mathrm{g} / \mathrm{ml}$ DIO-labeled H929 cell-derived exosomes for another $4 \mathrm{~h}$ in the presence of the indicated inhibitors. (C) The uptake was visualized by confocal microscopy and (D) the mean or median fluorescence intensity was determined by flow cytometry. ${ }^{* *} \mathrm{P}<0.01$ and ${ }^{* * * *} \mathrm{P}<0.001$, compared with $0 \mathrm{mM}$ amiloride/omeprazole. 


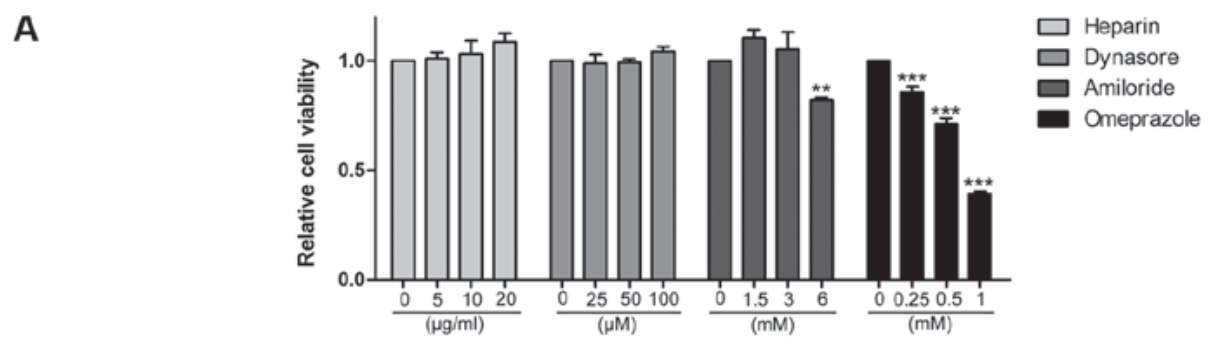

B
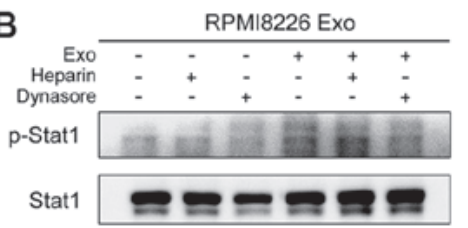

p-Stat3

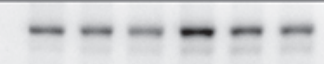

Stat3
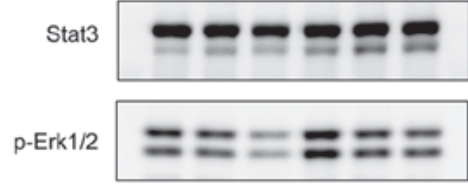

Erk1/2

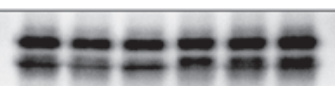

GAPDH

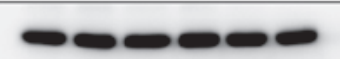

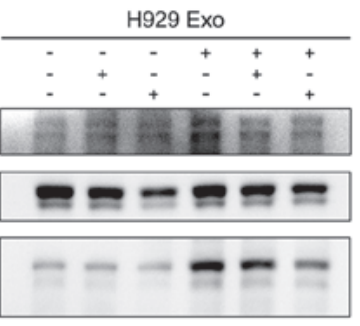
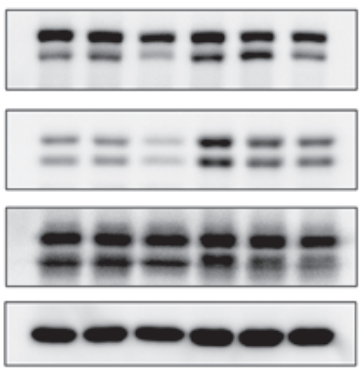

D

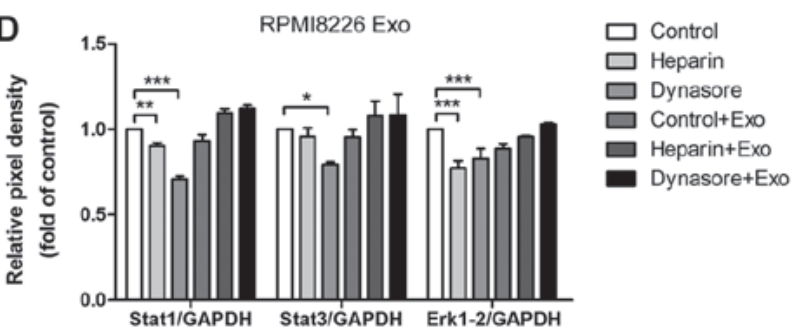

C
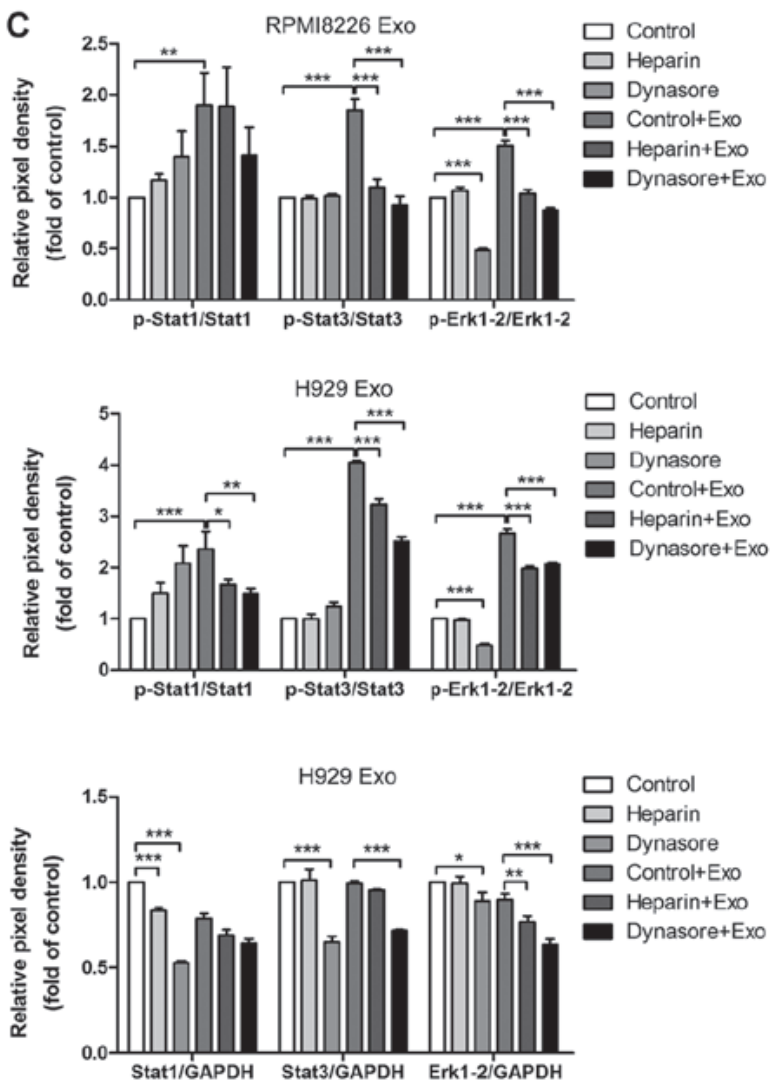

Figure 6. Endocytosis inhibitors partially suppress the effects of multiple myeloma cell-derived exosome on BMSCs. (A) Human BMSCs were incubated with heparin, dynasore, amiloride or omeprazole at the indicated final concentration for $4.5 \mathrm{~h}$, and cell viability was examined. ${ }^{* *} \mathrm{P}<0.01$ and ${ }^{* * *} \mathrm{P}<0.001$, compared with the group without inhibitor treatment. (B) Human BMSCs were pre-treated with heparin $(10 \mu \mathrm{g} / \mathrm{ml})$ or dynasore (50 $\mu \mathrm{M})$ for $30 \mathrm{~min}$ and incubated with $100 \mu \mathrm{g} / \mathrm{ml} \mathrm{RPMI8226}$ or H929 cell-derived exosomes for $6 \mathrm{~h}$. Subsequently, total and phosphorylated STAT1, STAT3 and ERK1/2 were quantified via western blot analysis. The GAPDH protein was included as a loading control. The pixel density of phosphorylated and total STAT1, STAT3 and ERK1/2 was quantified. (C) Phosphorylated proteins were normalized to their total protein amounts, and (D) total amounts of proteins were normalized to GAPDH quantities. "P $<0.05$; ${ }^{* *} \mathrm{P}<0.01$; and ${ }^{* * *} \mathrm{P}<0.001$. BMSCs, bone marrow stromal cells; Exo, exosomes; p, phosphor; STAT, signal transducer and activator of transcription 1; ERK1/2, extracellular signal-regulated kinase $1 / 2$.

sodium/proton exchanger, amiloride, dose-independently depressed exosomes internalization, and the exosomes uptake was reduced by $\sim 40 \%$ (Fig. 5A and B). Omeprazole, a proton pump inhibitor, dose-dependently attenuated exosomes uptake, and $1 \mathrm{mM}$ omeprazole decreased the mean fluorescence intensity in MSCs by up to $70 \%$, indicating strong inhibition (Fig. 5C and D). These results validate the involvement of macropinocytosis and membrane fusion in the uptake of MM cell-derived exosomes by BMSCs.

Endocytosis inhibitors suppress the effects of MMcell-derived exosomes on BMSCs. Although a reduction in exosomes internalization was observed in BMSCs treated with the inhibitors of endocytosis, macropinocytosis or membrane fusion, these inhibitors may influence the viability of BMSCs and therefore decrease exosomes uptake. Accordingly, the changes in BMSC viability following treatment with different inhibitors for $4 \mathrm{~h}$ at the concentration used in uptake experiments were evaluated (Fig. 6A). Heparin and dynasore did not impair BMSC viability, implying that the reduction of exosomes uptake by these two inhibitors is not caused by a decrease in BMSC viability. However, omeprazole and the highest dose of amiloride $(6 \mathrm{mM})$ significantly inhibited BMSC viability within $4 \mathrm{~h}$. Nonetheless, 1.5-3.0 mM amiloride, which reduced exosomes internalization, did not decrease BMSC viability, indicating that the inhibition of exosomes uptake induced by amiloride in this range of concentrations was not a result of impaired BMSC viability. Additionally, heparin and dynasore suppressed the phosphorylation of STAT1, STAT3, and ERK1/2 in the presence of RPMI8226 or H929 
exosomes, compared with treatment with RPMI8226 or H929 exosomes only (Fig. 6B-D), indicating that the suppression of exosomes uptake by these two inhibitors can attenuate the exosomes-induced functional changes in BMSCs.

\section{Discussion}

Accumulating evidence highlights EVs as crucial mediators in cell-to-cell communication during tumor progression, and a number of studies revealed that exosomes mediate the communication between BMSCs and MM cells, and promote the formation of a microenvironment with enhanced angiogenesis and immunosuppression $(22,32)$. Thus, blocking this communication may facilitate the treatment of MM, while the routes and mechanisms of the uptake of EVs involved in the MM cell-BMSC interaction remain unknown. Large macrovesicles (200-1,000 $\mathrm{nm}$ size) are also produced by tumor cells and meditate the communication between the tumor cells and stromal cells $(21,33)$. Unlike homogeneous exosomes, the shape of these vesicles is more irregular and inhomogeneous, resulting in difficulty in identifying them (34). Additionally, the release of large EVs is not constitutive and can be regulated by various stimuli and conditions, including starvation and cytokine stimulation (35). Thus, the present study primarily investigates the function of a subtype of homogeneous EVs, exosomes. In the present study, pharmacological inhibition of endocytic pathways was conducted and it was demonstrated that routes of uptake of MM cell-derived exosomes by BMSCs include caveolin-dependent endocytosis, macropinocytosis and membrane fusion. Furthermore, specific inhibitors suppressed the functional changes in BMSCs induced by the exosomes-mediated MM cell-to-BMSC communication. These data are indicative of a possible use of endocytic inhibitors as enhancers of MM treatment.

Previously, it was demonstrated that murine MM cell-derived exosomes carrying a variety of soluble factors, including monocyte chemoattractant protein 1 , macrophage inflammatory protein 1a and stromal cell-derived factor 1, can increase the viability of murine BMSCs and activate a number pro-survival or pro-proliferation pathways, including STAT3, JNK and p53, in BMSCs (22). To validate this observation in human cells, exosomes from four human MM cell lines were isolated, and all these exosomes promoted the viability of human BMSCs isolated from a healthy donor under starvation conditions. Similarly, MM cell-derived exosomes activated the STAT1, STAT3 and ERK1/2 pathways, which have been demonstrated to be associated with cell survival and proliferation (36-38). Additionally, our previous study indicated that miRNAs enclosed in MM cell-derived exosomes enhance the pro-tumoral activity of BMSCs (25), and these results highlight the involvement of exosomes in the functional regulation of target cells.

MM cell-derived exosomes can be time-dependently engulfed by BMSCs, and this process requires energy, in line with the observation reported in another study (39). Exosomes utilize native mechanisms of uptake, which consist of numerous endocytic pathways, including phagocytosis, clathrin- and caveolin-mediated endocytosis, to functionally deliver their cargo (8). Internalization of extracellular material is primarily subdivided into two subtypes, termed phagocytosis and pinocytosis (40). Large particles are primarily internalized through phagocytosis, and this process is generally restricted to phagocytes (41). In contrast, pinocytosis, including clathrin-dependent endocytosis, clathrin-independent endocytosis and macropinocytosis, can be conducted by all cell types to engulf relatively small vesicles and extracellular molecules $(42,43)$. Heparin functions as a soluble analogue of heparin sulphate proteoglycans and inhibits endocytosis (44). In the present study, heparin decreased the uptake of MM cell-derived exosomes by $\sim 78 \%$, indicating that endocytosis is the dominant route. Heparin has been reported to significantly inhibit the internalization of exosomes or EVs in numerous cell types, including glioblastoma multiform primary tumor cells, bladder cancer cells and human cervical carcinoma cells $(39,44)$. These studies highlight endocytosis as a primary route of exosomes entry into recipient cells.

Endocytosis can be categorized into clathrin-, flotillin-1-, RhoA- and caveolin-dependent endocytosis (45). Dynamin 2, a GTPase, is recruited to nascent clathrin-coated pits and is required during membrane fission and a release of clathrin-coated vesicles $(46,47)$. Dynamin 2 activity also promotes the assembly and expansion of caveolar vesicles (48). Suppression of dynamin 2 by a specific inhibitor (dynasore) can strongly reduce the internalization of exosomes $(39,49)$. Indeed, dynasore significantly decreased the uptake of MM cell-derived exosomes by $\sim 65 \%$ in the present study. The inhibitory effect of heparin was stronger than dynasore, indicating that other types of endocytosis are also involved in exosomes uptake. Treatment with chlorpromazine reduces uptake of EVs by ovarian cancer and phagocytic recipient cells $(31,50)$. By contrast, this inhibitor did not decrease the internalization of MM cell-derived exosomes in the present study, indicating that clathrin-mediated endocytosis does not contribute significantly to the aforementioned process. Thus, caveolin-dependent endocytosis is the primary endocytic pathway for the uptake of MM cell-derived exosomes by BMSCs.

Phagocytosis, macropinocytosis and membrane fusion also contribute to EV internalization. Although wortmannin has been reported to significantly inhibit EV uptake $(31,39)$, the present study did not reveal notable inhibitory effects on the uptake of MM cell-derived exosomes by BMSCs when wortmannin was used, indicating the absence of phagocytosis in this process. The first step of macropinocytosis is the formation of invagination-based membrane ruffles, and then they are pinched off into the intercellular space (8). Unlike phagocytosis, macropinocytosis does not require the direct contact with internalized materials but requires $\mathrm{Na}^{+} / \mathrm{H}^{+}$ exchanger activity $(14,51)$. Abrogation of macropinocytosis by amilorde, a $\mathrm{Na}^{+} / \mathrm{H}^{+}$exchanger inhibitor, reduces the $\mathrm{EV}$ internalization by microglia (49). The present results also uncovered an inhibitory effect of amilorde on the uptake of MM cell-derived exosomes by BMSCs, although this effect is not stronger than that induced by heparin and dynasore. These data indicate that caveolin-dependent endocytosis contributes more to the uptake than macropinocytosis. Direct fusion of the EV membrane with the cell membrane is another mechanism underlying exosomes uptake (3). Proton pump inhibitors have been demonstrated to decrease exosomes fusion with melanoma cells by targeting sodium reabsorption (16). Strong inhibition of exosomes uptake was observed in the present 
study in BMSCs treated with $1 \mathrm{mM}$ omeprazole, a proton pump inhibitor, whereas it decreased BMSC viability by $60 \%$. Lower concentrations of omeprazole still decreased BMSC viability, although omeprazole can inhibit exosomes uptake, indicating that this inhibition may be primarily caused by impaired cell viability instead of inhibition of membrane fusion. Furthermore, endocytosis inhibitors heparin and dynasore suppressed and delayed the phosphorylation of STAT1, STAT3, and ERK1/2 induced by MM cell-derived exosomes following a brief blockade. These results indicate that an endocytosis inhibitor can attenuate exosomes uptake and suppress exosomes-induced changes of intercellular pathways. These observations are indicative of potential usefulness of these inhibitors for blocking MM cell-BMSC communication. Nevertheless, further studies that reveal the specific ligand-receptor pairs responsible for exosomal uptake, as well as in vivo studies investigating the minimum effective dose range, are required in the future to improve the understanding of the uptake process and determine the blocking efficiency.

In conclusion, endocytosis was identified as the primary route of uptake of MM cell-derived exosomes by BMSCs and it was demonstrated that macropinocytosis and membrane fusion are also partially involved in exosomes internalization. These observations will facilitate the development of modification-based approaches to the enhancement of exosomes uptake and thus improve the efficiency of exosomes-mediated cargo or drug delivery. Additionally, the present data indicated that the blockade of the endocytosis pathway can suppress the exosomes-induced alteration in target BMSCs, underscoring the importance of this route in MM cell-BMSC communication. Furthermore, blocking the communication between MM cells and BMSCs may be another option for improving MM treatment due to this communication being demonstrated to be critical for MM progression.

\section{Acknowledgements}

The authors would like to thank Mr. Yonghua Liao at School of Basic Medical Sciences, Guangzhou Medical University (Guangzhou, China) for expert technical assistance.

\section{Funding}

The present study was supported by the National Natural Science Foundation of China (grant no. 81700203), Medical Scientific Research Foundation of Guangdong Province, China (project no. A2018031) and startup funding issued by Guangzhou Medical University and Bureau of Education of Guangzhou.

\section{Availability of data and materials}

All data and materials in this study are available from corresponding author on reasonable request.

\section{Authors' contributions}

JW conceived the idea and supervised the experiments. YZ and CT implemented the experiments with the assistance of
JZ. JW, YZ and CT analyzed the data and wrote the manuscript with suggestions from all the authors. All the authors read and approved the final manuscript.

\section{Ethics approval and consent to participate}

Ethical approval was obtained from Ethical Committee for Clinical Medicine Research of The Third Affiliated Hospital of Sun Yat-Sen University (Guangzhou, China). Informed consent was obtained from participate prior to sample collection.

\section{Patient consent for publication}

Consent for publication was obtained from the participants.

\section{Competing interests}

The authors declare that they have no competing interests.

\section{References}

1. Tkach M and Théry C: Communication by extracellular vesicles: Where we are and where we need to go. Cell 164: 1226-1232, 2016.

2. Camussi G, Deregibus MC, Bruno S, Grange C, Fonsato V and Tetta C: Exosome/microvesicle-mediated epigenetic reprogramming of cells. Am J Cancer Res 1: 98-110, 2011.

3. Raposo G and Stoorvogel W: Extracellular vesicles: Exosomes, microvesicles, and friends. J Cell Biol 200: 373-383, 2013.

4. Théry C, Zitvogel L and Amigorena S: Exosomes: Composition, biogenesis and function. Nat Rev Immunol 2: 569-579, 2002.

5. Ratajczak J, Wysoczynski M, Hayek F, Janowska-Wieczorek A and Ratajczak MZ: Membrane-derived microvesicles: Important and underappreciated mediators of cell-to-cell communication. Leukemia 20: 1487-1495, 2006.

6. EL Andaloussi S, Mäger I, Breakefield XO and Wood MJ; S ELA: Extracellular vesicles: Biology and emerging therapeutic opportunities. Nat Rev Drug Discov 12: 347-357, 2013.

7. Colombo M, Raposo G and Théry C: Biogenesis, secretion, and intercellular interactions of exosomes and other extracellular vesicles. Annu Rev Cell Dev Biol 30: 255-289, 2014.

8. Mulcahy LA, Pink RC and Carter DR: Routes and mechanisms of extracellular vesicle uptake. J Extracell Vesicles 3: 3, 2014

9. O'Donoghue EJ and Krachler AM: Mechanisms of outer membrane vesicle entry into host cells. Cell Microbiol 18: 1508-1517, 2016

10. Christianson HC, Svensson KJ, van Kuppevelt TH, Li JP and Belting M: Cancer cell exosomes depend on cell-surface heparan sulfate proteoglycans for their internalization and functional activity. Proc Natl Acad Sci USA 110: 17380-17385, 2013.

11. Ehrlich M, Boll W, Van Oijen A, Hariharan R, Chandran K, Nibert ML and Kirchhausen T: Endocytosis by random initiation and stabilization of clathrin-coated pits. Cell 118: 591-605, 2004.

12. Stephens L, Ellson C and Hawkins P: Roles of PI3Ks in leukocyte chemotaxis and phagocytosis. Curr Opin Cell Biol 14: 203-213, 2002.

13. Wang LH, Rothberg KG and Anderson RG: Mis-assembly of clathrin lattices on endosomes reveals a regulatory switch for coated pit formation. J Cell Biol 123: 1107-1117, 1993.

14. Swanson JA: Shaping cups into phagosomes and macropinosomes. Nat Rev Mol Cell Biol 9: 639-649, 2008.

15. Koivusalo M, Welch C, Hayashi H, Scott CC, Kim M, Alexander T, Touret N, Hahn KM and Grinstein S: Amiloride inhibits macropinocytosis by lowering submembranous $\mathrm{pH}$ and preventing Rac1 and Cdc42 signaling. J Cell Biol 188: 547-563, 2010.

16. Parolini I, Federici C, Raggi C, Lugini L, Palleschi S, De Milito A, Coscia C, Iessi E, Logozzi M, Molinari A, et al: Microenvironmental $\mathrm{pH}$ is a key factor for exosome traffic in tumor cells. J Biol Chem 284: 34211-34222, 2009.

17. Ha D, Yang N and Nadithe V: Exosomes as therapeutic drug carriers and delivery vehicles across biological membranes: Current perspectives and future challenges. Acta Pharm Sin B 6: 287-296, 2016. 
18. Batrakova EV and Kim MS: Using exosomes, naturally-equipped nanocarriers, for drug delivery. J Control Release 219: 396-405, 2015.

19. Lemaire M, Deleu S, De Bruyne E, Van Valckenborgh E, Menu E and Vanderkerken K: The microenvironment and molecular biology of the multiple myeloma tumor. Adv Cancer Res 110: 19-42, 2011.

20. Manier S, Sacco A, Leleu X, Ghobrial IM and Roccaro AM: Bone marrow microenvironment in multiple myeloma progression. J Biomed Biotechnol 2012: 157496, 2012.

21. Wang J, Faict S, Maes K, De Bruyne E, Van Valckenborgh E, Schots R, Vanderkerken K and Menu E: Extracellular vesicle cross-talk in the bone marrow microenvironment: Implications in multiple myeloma. Oncotarget 7: 38927-38945, 2016.

22. Wang J, De Veirman K, Faict S, Frassanito MA, Ribatti D, Vacca A and Menu E: Multiple myeloma exosomes establish a favourable bone marrow microenvironment with enhanced angiogenesis and immunosuppression. J Pathol 239: 162-173, 2016.

23. Wang J, De Veirman K, De Beule N, Maes K, De Bruyne E, Van Valckenborgh E, Vanderkerken K and Menu E: The bone marrow microenvironment enhances multiple myeloma progression by exosome-mediated activation of myeloid-derived suppressor cells. Oncotarget 6: 43992-44004, 2015.

24. Wang J, Hendrix A, Hernot S, Lemaire M, De Bruyne E, Van Valckenborgh E, Lahoutte T, De Wever O, Vanderkerken K and Menu E: Bone marrow stromal cell-derived exosomes as communicators in drug resistance in multiple myeloma cells. Blood 124: 555-566, 2014.

25. De Veirman K, Wang J, Xu S, Leleu X, Himpe E, Maes K, De Bruyne E, Van Valckenborgh E, Vanderkerken K, Menu E, et al: Induction of miR-146a by multiple myeloma cells in mesenchymal stromal cells stimulates their pro-tumoral activity. Cancer Lett 377: 17-24, 2016.

26. Zuhorn IS, Kalicharan R and Hoekstra D: Lipoplex-mediated transfection of mammalian cells occurs through the cholesterol-dependent clathrin-mediated pathway of endocytosis. J Biol Chem 277: 18021-18028, 2002

27. Uhrig S, Coutelle O, Wiehe T, Perabo L, Hallek M and Büning H: Successful target cell transduction of capsid-engineered rAAV vectors requires clathrin-dependent endocytosis. Gene Ther 19: 210-218, 2012.

28. Kim KS, Yoon YR, Lee HJ, Yoon S, Kim SY, Shin SW, An JJ, Kim MS, Choi SY, Sun W, et al: Enhanced hypothalamic leptin signaling in mice lacking dopamine D2 receptors. J Biol Chem 285: 8905-8917, 2010.

29. Gawecka JE, Young-Robbins SS, Sulzmaier FJ, Caliva MJ, Heikkilä MM, Matter ML and Ramos JW: RSK2 protein suppresses integrin activation and fibronectin matrix assembly and promotes cell migration. J Biol Chem 287: 43424-43437, 2012.

30. MacGibeny MA, Koyuncu OO, Wirblich C, Schnell MJ and Enquist LW: Retrograde axonal transport of rabies virus is unaffected by interferon treatment but blocked by emetine locally in axons. PLoS Pathog 14: e1007188, 2018.

31. Feng D, Zhao WL, Ye YY, Bai XC, Liu RQ, Chang LF, Zhou Q and Sui SF: Cellular internalization of exosomes occurs through phagocytosis. Traffic 11: 675-687, 2010.

32. Umezu T, Tadokoro H, Azuma K, Yoshizawa S, Ohyashiki K and Ohyashiki JH: Exosomal miR-135b shed from hypoxic multiple myeloma cells enhances angiogenesis by targeting factor-inhibiting HIF-1. Blood 124: 3748-3757, 2014.
33. Théry C, Ostrowski $M$ and Segura E: Membrane vesicles as conveyors of immune responses. Nat Rev Immunol 9: 581-593, 2009.

34. Raimondo F, Morosi L, Chinello C, Magni F and Pitto M: Advances in membranous vesicle and exosome proteomics improving biological understanding and biomarker discovery. Proteomics 11: 709-720, 2011.

35. Orozco AF and Lewis DE: Flow cytometric analysis of circulating microparticles in plasma. Cytometry A 77: 502-514, 2010.

36. Meissl K, Macho-Maschler S, Müller M and Strobl B: The good and the bad faces of STAT1 in solid tumours. Cytokine 89: 12-20, 2017

37. WingelhoferB, NeubauerHA, Valent P,HanX,Constantinescu SN Gunning PT, Müller M and Moriggl R: Implications of STAT3 and STAT5 signaling on gene regulation and chromatin remodeling in hematopoietic cancer. Leukemia 32: 1713-1726, 2018.

38. Tanimura S and Takeda K: ERK signalling as a regulator of cell motility. J Biochem 162: 145-154, 2017.

39. Costa Verdera H, Gitz-Francois JJ, Schiffelers RM and Vader P: Cellular uptake of extracellular vesicles is mediated by clathrin-independent endocytosis and macropinocytosis. J Controll Release 266: 100-108, 2017.

40. Doherty GJ and McMahon HT: Mechanisms of endocytosis. Annu Rev Biochem 78: 857-902, 2009.

41. Rosales C and Uribe-Querol E: Phagocytosis: A fundamental process in immunity. BioMed Res Int 2017: 9042851, 2017.

42. Kaksonen M and Roux A: Mechanisms of clathrin-mediated endocytosis. Nat Rev Mol Cell Biol 19: 313-326, 2018.

43. Rosendale $M$ and Perrais D: Imaging in focus: Imaging the dynamics of endocytosis. Int J Biochem Cell Biol 93: 41-45, 2017.

44. Franzen CA, Simms PE, Van Huis AF, Foreman KE, Kuo PC and Gupta GN: Characterization of uptake and internalization of exosomes by bladder cancer cells. BioMed Res Int 2014: 619829, 2014.

45. Mayor S and Pagano RE: Pathways of clathrin-independent endocytosis. Nat Rev Mol Cell Biol 8: 603-612, 2007.

46. Taylor MJ, Lampe M and Merrifield CJ: A feedback loop between dynamin and actin recruitment during clathrin-mediated endocytosis. PLoS Biol 10: e1001302, 2012.

47. Marks B, Stowell MH, Vallis Y, Mills IG, Gibson A, Hopkins CR and McMahon HT: GTPase activity of dynamin and resulting conformation change are essential for endocytosis. Nature 410: 231-235, 2001.

48. Parton RG and Simons K: The multiple faces of caveolae. Nat Rev Mol Cell Biol 8: 185-194, 2007.

49. Fitzner D, Schnaars M, van Rossum D, Krishnamoorthy G, Dibaj P, Bakhti M, Regen T, Hanisch UK and Simons M: Selective transfer of exosomes from oligodendrocytes to microglia by macropinocytosis. J Cell Sci 124: 447-458, 2011.

50. Escrevente C, Keller S, Altevogt P and Costa J: Interaction and uptake of exosomes by ovarian cancer cells. BMC Cancer 11: $108,2011$.

51. Kerr MC and Teasdale RD: Defining macropinocytosis. Traffic 10: 364-371, 2009 